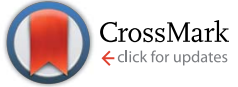

Received 14th November 2016 Accepted 4th January 2017

DOI: 10.1039/c6ra26753j

www.rsc.org/advances
Cite this: RSC Adv., 2017, 7, 5177

\section{Facile fabrication of nanoporous graphene powder for high-rate lithium-sulfur batteries $\uparrow$}

\author{
Huajie Zhuang, ${ }^{\text {ab }}$ Wei Deng, ${ }^{a}$ Wei Wang ${ }^{\star a}$ and Zhaoping Liu ${ }^{\star a}$
}

Well-designed structures constructed from graphene are excellent sulfur host matrices which can improve the electrochemical performance of lithium-sulfur $(\mathrm{Li}-\mathrm{S})$ batteries by alleviating the dissolution of polysulfide and improving the electrical conductivity of the electrode. Herein, high quality graphene powder with a nanoshell inside was successfully designed and fabricated through a simple spray-drying and Fe-catalyzed chemical vapor deposition (CVD) process. In this structure, the intrinsic interconnected graphene nanoshells afford sufficient space for accommodating the active sulfur material, provide effective entrapment for the dissolution of polysulfide and facilitate fast electron and mass transport. Benefiting from this unique architecture, a high specific discharge capacity of $400 \mathrm{~mA} \mathrm{~h} \mathrm{~g} \mathrm{~g}^{-1}$ with wellmaintained two-step galvanostatic discharge profiles can be obtained at an ultra-high current density of $13.4 \mathrm{~A} \mathrm{~g}^{-1}$.

\section{Introduction}

With the high-speed development of electrical vehicles and portable electronic products, there is tremendous demand for high energy density and high power density energy storage devices. Lithium-sulfur (Li-S) batteries, which have been known and investigated since the 1970s, are considered as promising candidates for next-generation energy storage devices owing to their distinguished advantages including high theoretical energy density $\left(1675 \mathrm{~mA} \mathrm{~h} \mathrm{~g}^{-1}\right)$, and being abundant sources in nature, economical and environment friendly materials. However, their practical applications have been plagued by the dissolution of polysulfide, insulation of sulfur (5 $\times 10^{-30} \mathrm{~S} \mathrm{~cm}^{-1}$ ) and considerable volume change of sulfur during electrochemical cycling processes, which results in a series of serious problems, such as low specific discharge capacity, severe self-charge, overcharging and short cycling performance..$^{-4}$

During past several decades, many efforts have been made to alleviate above problems. To fully enable the electrochemical performance of active sulfur in Li-S batteries, the host materials for sulfur play the significantly important role, which should have sufficient specific surface area to ensure high sulfur loading, a conductive barrier to suppress the dissolution of

${ }^{a}$ Ningbo Institute of Materials Technology and Engineering, Chinese Academy of Sciences, Ningbo, Zhejiang 315201, P. R. China. E-mail: wangwei@nimte.ac.cn; liuzp@nimte.ac.cn

${ }^{b}$ Nano Science and Technology Institute, University of Science and Technology of China, P. R. China

$\dagger$ Electronic supplementary information (ESI) available: SEM image of NGP-SiO NGP and DGP. TEM image of DGP. Nitrogen adsorption-desorption isotherms and pore size of DGP. TG curve of NGP/S. See DOI: 10.1039/c6ra26753j polysulfide and facilitate the electrical transport, and also a porous channel for the effective infiltration of electrolyte and transfer of Li-ion. Meeting well with the above requirements, porous carbon has been proved to be one of the most promising sulfur host materials. ${ }^{3,4}$ Pioneering work carried out by Nazar's group successfully developed CMK-3 type mesoporous carbon with a polymer coating which greatly alleviated dissolution of polysulfide for achieving significantly enhanced battery performance. ${ }^{5}$ After that, many researches have been focused on the search of advanced carbon for Li-S batteries, such as porous carbon, ${ }^{6-8}$ carbon shell, ${ }^{9-11}$ carbon nanofiber, ${ }^{12,13}$ carbon nanotube ${ }^{14-16}$ etc. For example, meso-microporous core-shell carbon structure was designed and prepared as sulfur container, in which the mesoporous core ensured sufficient sulfur loading and the microporous shell acted as a physical barrier for polysulfide for reaching long cycling performance and high discharge capacity. ${ }^{6}$ Guan et al. reported a tube-in-tube structure to host sulfur by encapsulating MWNTs into hollow porous carbon nanotubes that the outstanding electrochemical performance was obtained owing to unique structure with the high electrical conductivity, large pore volume and pore carbon layers. ${ }^{17}$ Many other well-designed porous structures, such pielike, ${ }^{18}$ cauliflower-like, ${ }^{19}$ and laminated structure ${ }^{20}$ were also built for lithium-sulfur batteries and had been proved to be efficient to alleviate the dissolution of polysulfide and improve the electrical conductivity of cathode.

More recently, as one kind of super-carbon materials, graphene presents the promising candidate for Li-S battery applications and other energy storage devices ${ }^{21-26}$ owing to the ultra-high conductivity, large specific surface area and sturdy mechanical properties. Self-assembled reduced graphene oxide (rGO) is most widely used as sulfur host due to the simply 
constructed three-dimensional (3D) structure and tunable pore size. ${ }^{27-30}$ For example, Li et al. had reported a 3D graphene hybrid sponges (3D-GHS) with pore size of about several microns by assembling $\mathrm{rGO}$ and GHS/sulfur which exhibited a high areal specific capacitance of $6.0 \mathrm{~mA} \mathrm{~h} \mathrm{~cm}{ }^{-2}$ after 11 cycles and retained capacity of $4.2 \mathrm{~mA} \mathrm{~h} \mathrm{~cm}{ }^{-2}$ after 300 cycles. $^{30}$ Even with advantages of facile fabrication and high yield by no matter the modified Hummer's method or exfoliation method, however the resulted graphene usually is of unavoidable defects which lead to a serious loss of electrical conductivity. Moreover, it has also indicated that chemical reactions between lithium and $\mathrm{rGO}$ with the formation of $\mathrm{Li}_{2} \mathrm{CO}_{3}, \mathrm{Li}_{2} \mathrm{SO}_{3}, \mathrm{Li}_{2} \mathrm{SO}_{4}$ and $\mathrm{CoSO}_{2} \mathrm{Li}$ species can lead to loss of recyclable active sulfur and hence the degradation of specific discharge capacity. ${ }^{31}$ Among various fabrication methods of graphene, chemical vapor deposition (CVD) is one of widely accepted approaches which can obtain high-quality graphene with high electrical conductivity. ${ }^{32} 3 \mathrm{D}$ porous graphene network prepared via CVD has been widely used in the field of energy storage, such as supercapacitors, Li-ion batteries and lithium-sulfur batteries. ${ }^{33-36}$ Kumar et al. fabricated a three-dimensional few-layer graphene foam by CVD method for Li-S battery application that excellent electrochemical stability and high rate performance were obtained owing to conductive interconnected foam with appropriate pore to contain sulfur and entrap dissolution of polysulfide. ${ }^{36}$

However, graphene obtained from $\mathrm{Ni}$ foam and other plane template is usually of low quantity, low volumetric density and also high cost, which was quite limited for Li-S battery application. A facile approach to obtain both high-quality and highquantity graphene powder through CVD process is required. It is reported that graphene prepared by CVD with solid carbon source was considered low cost and practical application. ${ }^{37,38}$ In this study, we developed a facile method to prepare high quantity graphene powder with nanoshells inside through spray drying and subsequently CVD process with solid carbon source. After permeating sulfur into NGP via a sulfur solution infiltration method, excellent electrochemical performance can be expected due to the unique architecture which afford sufficient space for accommodating the active sulfur material, provide effective entrapment for dissolution of polysulfide and facilitate the fast electron and mass transport.

\section{Experimental}

A typical fabrication process of NGP is shown in Fig. $1.12 .8 \mathrm{~g}$ PVA (Aladdin Co., Ltd, 87.0-89.0\%) was dissolved in $500 \mathrm{~mL}$ deionized (DI) water at $90{ }^{\circ} \mathrm{C}$ (solution A), and $7.6 \mathrm{~g} \mathrm{FeCl}$ (Aladdin Co., Ltd, 99.9\%) together with $6.4 \mathrm{~g} \mathrm{SiO}_{2}$ (Taijihuan Co., Ltd, $\mathrm{SiO}_{2}-1$ ) were dispersed in $300 \mathrm{~mL}$ DI water (solution $\mathrm{B}$ ). These two kinds of as-obtained solution were mixed together with stirring for 2 hours and following ultrasonication for 30 minutes (solution C). Amounts of powder could be collected by spray drying the solution $\mathrm{C}$ at $220^{\circ} \mathrm{C}$. After that, the powder was placed in the center of a quartz tube with diameter of $5 \mathrm{~cm}$ and length of $120 \mathrm{~cm}$ and then heated at $1000{ }^{\circ} \mathrm{C}$ for 30 minutes with a continuous flow of $50 \mathrm{sccm} \mathrm{H}_{2}$ and $100 \mathrm{sccm} \mathrm{Ar}$ at a pressure

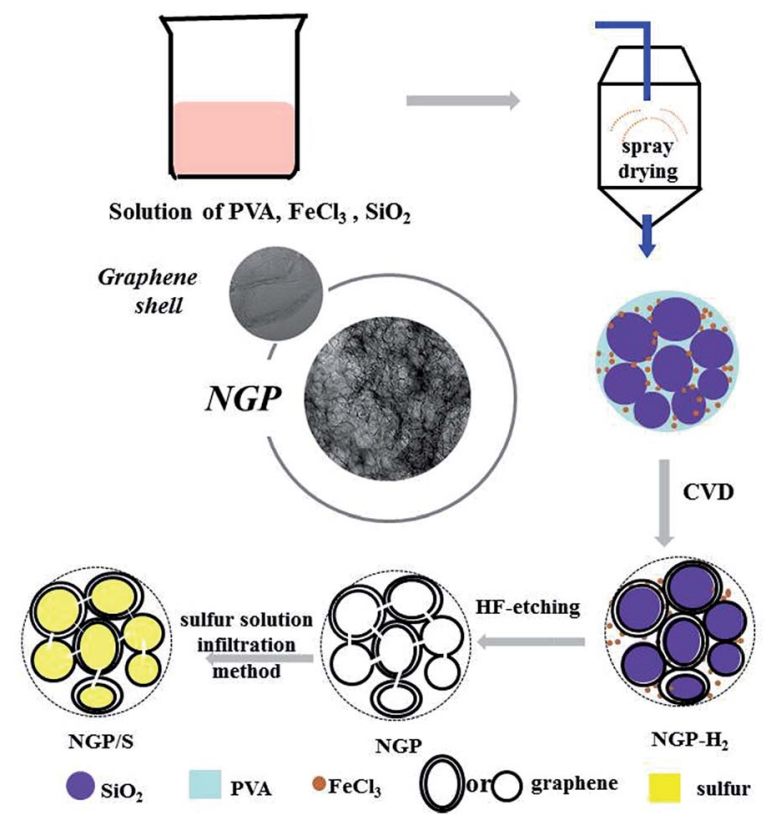

Fig. 1 Schematic illustration of the process for preparing NGP/S composites.

of $10 \mathrm{~Pa}$. Finally, the NGP powder was obtained by removing of $\mathrm{Fe}$ and $\mathrm{SiO}_{2}$ with $\mathrm{HF}$ solution. As a comparison, dense graphene powder (DGP) without pores inside was also prepared using the similar process without the introduction of $\mathrm{SiO}_{2}$ in solution.

For the Li-S battery applications, the active material sulfur (Aladdin Co., Ltd, 99.95\%) was loaded into NGP and DGP. NGP or DGP with weight of $0.1 \mathrm{~g}$ was placed in a glass bottle in size of $12 \mathrm{~mL}$, and then $3 \mathrm{~mL}$ carbon disulfide $\left(\mathrm{CS}_{2}\right.$, Sinopharm Chemical Regent Co., Ltd, 99.9\%) with 0.15 g sulfur was dropped inside. After the $\mathrm{CS}_{2}$ volatilized, the bottle was sealed and placed in an oven, heated to $155{ }^{\circ} \mathrm{C}$ and maintained for 12 hours.

The morphology was characterized by a Hitachi S-4800 field emission scanning electrical microscope (FE-SEM) and an FEI Tecnai G2 F20 transmission electrical microscopy (TEM). Thermogravimetric analysis (TGA) was performed on TGA50 analyzer (Shimadzu) under $\mathrm{N}_{2}$ atmosphere with a heating rate of $10{ }^{\circ} \mathrm{C} \mathrm{min}^{-1}$. Structure was identified by the X-ray diffraction (XRD) patterns characterization using an AXS D8 Advance Diffractometer $(\mathrm{Cu} \mathrm{K} \alpha$ radiation; receiving slit, $0.2 \mathrm{~mm}$; scintillation counter; $40 \mathrm{~mA}, 40 \mathrm{kV})$. The nitrogen sorption isotherms were measured by Micromeritics ASAP-2020M nitrogen adsorption apparatus. The pore size distribution plot was obtained by Barrett-Joyner-Halenda (BJH).

The working electrode was consisted of $80 \mathrm{wt} \%$ of active material NGP/S, $10 \mathrm{wt} \%$ of polyvinylidene fluoride (PVDF) as adhesion agents, $6 \mathrm{wt} \%$ of super-P (Shanghai hui industrial chemical Co., Ltd) conductive carbon and $4 \mathrm{wt} \%$ of vapor grow carbon fiber (VGCF, Showa Denko, VGCF-H). After stirring for 12 hours, the slurry was casted onto aluminum foil and dried at $80{ }^{\circ} \mathrm{C}$ for 12 hours. The as-prepared electrode was then assembled into 2032-type coin cells with Li metal foil as counter electrode. The electrolyte contained $1.0 \mathrm{M}$ lithium 
bistrifluoromethanesulfonylimide (LTFSI, Alfa aesar Co., Ltd, 99.99\%) in 1,3-dioxolane (Sinopharm Chemical Regent Co., Ltd, 97\%) and 1,2-dimethoxyethane (Sinopharm Chemical Regent Co., Ltd, 99.5\%) (volume ratio $1: 1$ ), and $0.1 \mathrm{M}$ lithium nitrate (Aladdin Co., Ltd, 99.99\%) was added as additive. A LANDCT2001A battery test system (Jinnuo Wuhan Corp., china) was used to test the electrochemical performance. Cyclic voltammetry measurement was performed at a scan rate of $0.1 \mathrm{mV} \mathrm{s}^{-1}$ using an electrochemical workstation (Autolab 83710). The current density varied from $0.2 \mathrm{C}$ to $8 \mathrm{C}\left(1 \mathrm{C}=1675 \mathrm{~mA} \mathrm{~g}^{-1}\right)$ with a cutoff voltage of 1.6-2.8 V.

\section{Results and discussion}

The typical preparation process of NGP/S was illustrated in Fig. 1. In this method, $\mathrm{FeCl}_{3}$ can be embedded in PVA with various $\mathrm{SiO}_{2}$ nanoparticles around by spray-drying process. During the subsequent heating process, the $\mathrm{FeCl}_{3}$ precursor will be turned to Fe which serves as the embedded catalyst for growth of graphene during PVA decomposition process. Meanwhile, abundant nanopores can be generated inside the graphene powder by etching $\mathrm{SiO}_{2}$ nanoparticles with $\mathrm{HF}$ solution. Finally, abundant NGP powder in size of $10-20 \mu \mathrm{m}$ can be collected, as shown in Fig. S1. $\dagger$ Typically, $1.1 \mathrm{~g}$ NGP can be collected by spray drying $800 \mathrm{~mL}$ solution with $12.8 \mathrm{~g}$ PVA contained, and the quantity can be easily increased by increasing the precursor solution.

As shown in Fig. 2a, the as-prepared NGP particles is in shape of microsized spheres with diameter of $10-20 \mu \mathrm{m}$, which is similar to morphology of original particles just after spray drying process (Fig. S1†). However, with further observation under TEM in Fig. 2b, numerous pores appear inside the spheres after the HF etching process. HRTEM result in Fig. 2c further demonstrates that the pore size is about $20-30 \mathrm{~nm}$ which consist with the particle diameter of purchased $\mathrm{SiO}_{2}$. Moreover, it is demonstrated that the wall of shell was constructed by few-layer graphene as clear layer structures within ten layer with a layer spacing of $0.35 \mathrm{~nm}$ was observed. ${ }^{32,39}$

The contrast samples were tried without the addition of $\mathrm{FeCl}_{3}$ or $\mathrm{SiO}_{2}$. However, there was almost no collection of powder after CVD process if $\mathrm{FeCl}_{3}$ was not used in the fabrication precursor. The carbon decomposed from PVA might be etched during this process, which demonstrates the important role of Fe for effectively absorbing carbon source and resulting in the formation of few-layer graphene around. On the other hand, a significantly different morphology was observed in DGP sample without $\mathrm{SiO}_{2}$. As shown in Fig. 2d, the surface of DGP is much smoother and denser. Compared to the porous structure in NGP sample, no pore is obviously observed in Fig. 2e. As shown in Fig. 2f, DGP is also constructed by graphitic layer. As shown in Fig. S2a and b, $\dagger$ the SSA of DGP is about $90 \mathrm{~cm}^{2} \mathrm{~g}^{-1}$, and only pore peak at about $4 \mathrm{~nm}$ is observed. It can be confirmed that the abundant nanopores were mainly originate from $\mathrm{SiO}_{2}$. As $\mathrm{SiO}_{2}$ nanoparticles can be produced in large quantity in a broad size range of a few nanometer to micrometers, it can be inferred that the pore size can be tunned by $\mathrm{SiO}_{2}$.

The resulted hollow spheres provided the ideal spaces for the accommodation of active sulfur. As shown in Fig. $2 \mathrm{~g}$, no obvious
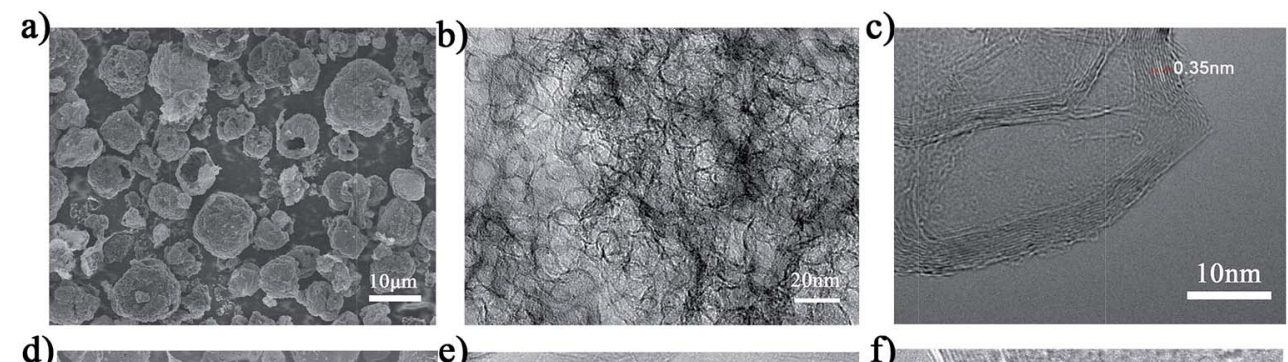

d)
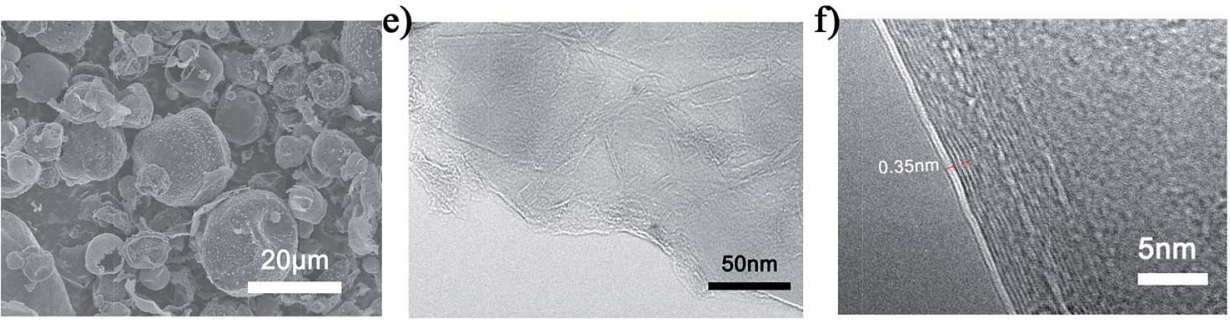

g)
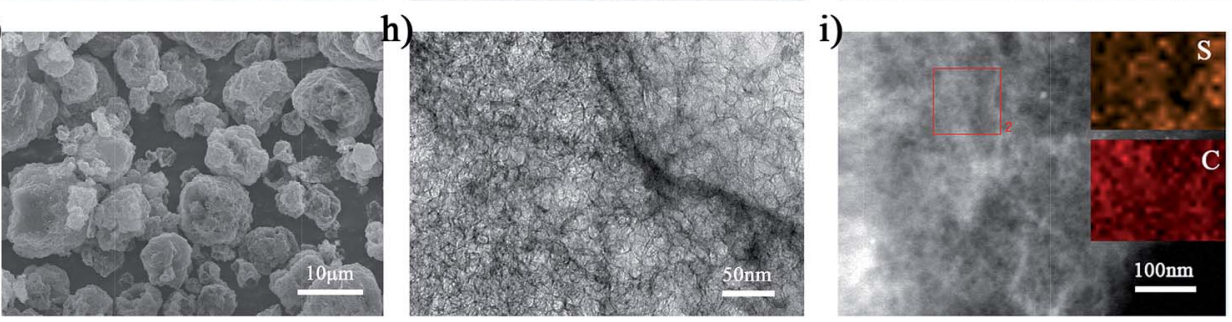

Fig. 2 (a) SEM image, (b) TEM image and (c) high-resolution TEM image of NGP. (d) SEM image, (e) TEM image and (f) high-resolution TEM image of DGP. (g) SEM and (h) TEM image of NGP/S. (i) Energy dispersive spectroscopy (EDS) of NGP/S. 
sulfur particle is observed on surface after infiltrating sulfur into NGP. The as-obtained NGP/S remains the same morphology which suggesting strong mechanical properties of graphene structure during the fabrication process. The distribution of sulfur in the host materials had a notable influence on electrochemical properties. The uniform distribution in the designed structure was crucial to the utilization of sulfur and specific capacity. TEM result in Fig. $2 \mathrm{~h}$ also confirms that unordered pores can be briefly observed without any obvious sulfur particles appearing which indicates the homogenous infiltration of sulfur into the graphene nanoshell inside NGP. With further EDS mapping analysis in Fig. 2i, it is found that the sulfur was homogenously mixed with porous few layer graphene.

To evaluate the crystallographic structure of the as-prepared NGP, the characterization of XRD and Raman were made. As shown in Raman spectra in Fig. 3a, three featured peaks of Dband, G-band and 2D-band of graphite at 1350, 1580 and $2700 \mathrm{~cm}^{-1}$ are observed. The D band represents defects of graphene and G-band corresponds to the vibration of $\mathrm{sp}^{2}$ banded carbon atoms in the graphite lattice. Compared to the Raman spectra in reduced graphene oxide via a routine method, NGP exhibits the lower D band and sharper 2D band, which demonstrates the higher quality of CVD-grown nanoporous graphene. As shown in Fig. 3b, XRD peaks centered at $2 \theta$ of $\sim 26^{\circ}$ and $\sim 44^{\circ}$ can be indexed to (002) and (101) crystal planes of graphite (JCPDS-01-075-1621). As indicated in Fig. 3b, the sharp peak of sulfur is almost negligible in the NGP/S sample which suggests that sulfur has been homogenously infused into the hollow sphere. The sulfur content can be examined by TGA characterization. As shown in Fig. S3, $\dagger$ with the temperature range of from $25{ }^{\circ} \mathrm{C}$ to $600{ }^{\circ} \mathrm{C}$, an obvious weight loss happened
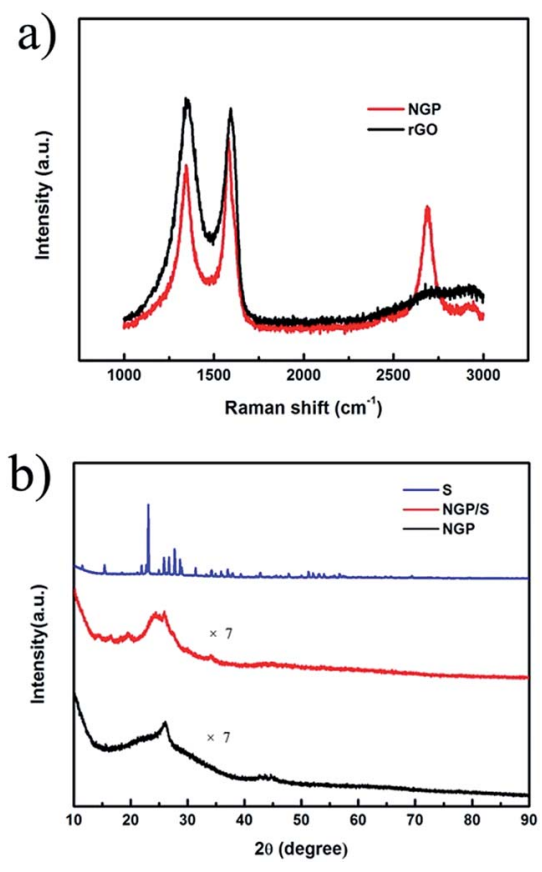

Fig. 3 (a) Raman spectra of NGP and rGO. (b) XRD pattern of NGP. at between 200 and $300{ }^{\circ} \mathrm{C}$, which was related to a sulfur content of about $60 \mathrm{wt} \%$. In a overall consideration, the sulfur content was usually controlled about $60 \%$ for must sulfur host materials. ${ }^{39-41}$

The structure evolution can also be presented by the change of SSA and pore size. For $\mathrm{NGP}_{-} \mathrm{SiO}_{2}$ sample just after CVD process without etching $\mathrm{SiO}_{2}$ and Fe particles, SSA is $74 \mathrm{~m}^{2} \mathrm{~g}^{-1}$ and no pore is clearly observed as shown in Fig. 4. After removal of $\mathrm{Fe}$ and $\mathrm{SiO}_{2}$, SSA abruptly increases to $728 \mathrm{~m}^{2} \mathrm{~g}^{-1}$ and simultaneously three significant peaks at $4 \mathrm{~nm}, 10 \mathrm{~nm}$ and $30 \mathrm{~nm}$ appear in NGP, which consists well with the nanopores observed in Fig. 2a and b. The pore volume of NGP is $2.5 \mathrm{~m}^{3} \mathrm{~g}^{-1}$. The high SSA and mesopores provide capability of high sulfur loading and tolerance of the volume expansion. After the sulfur infiltration process, SSA of NGP/S recovers to the $26 \mathrm{~cm}^{2} \mathrm{~g}^{-1}$ and the pores at 4, 10, $30 \mathrm{~nm}$ are vanished, which strongly demonstrates that sulfur has been filled into the hollow shell. ${ }^{39}$

The electrochemical properties of NGP/S have been investigated in detail as shown in Fig. 5. The half-cell was cycled in a voltage window of $1.6-2.8 \mathrm{~V}$ and the specific discharge capacity was calculated based on the mass of sulfur. The areal mass loading of active material was about $2.0 \mathrm{mg} \mathrm{cm}^{-2}$ and the thickness of active material on aluminum foil was about $20 \mu \mathrm{m}$ after pressing under $50 \mathrm{MPa}$. The cyclic voltammogram of NGP/ $\mathrm{S}$ at the scan rate of $0.1 \mathrm{mV} \mathrm{s}^{-1}$ from first to fifth cycle was presented in Fig. 5a. Two cathodic peaks at $2.28 \mathrm{~V}$ and $2.01 \mathrm{~V}$ appear which are ascribed to the reduction from elemental sulfur to lithium polysulfide and further to lithium sulfides respectively. The anodic peaks at $2.35 \mathrm{~V}$ and $2.43 \mathrm{~V}$ correspond
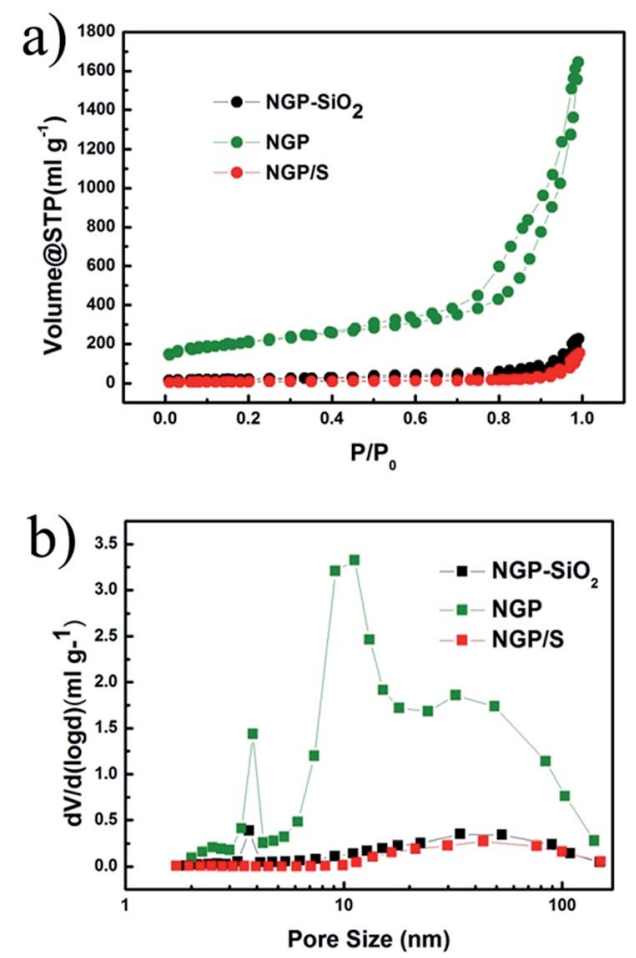

Fig. 4 (a) Nitrogen adsorption-desorption isotherms and (b) corresponding pore size distribution of NGP. 

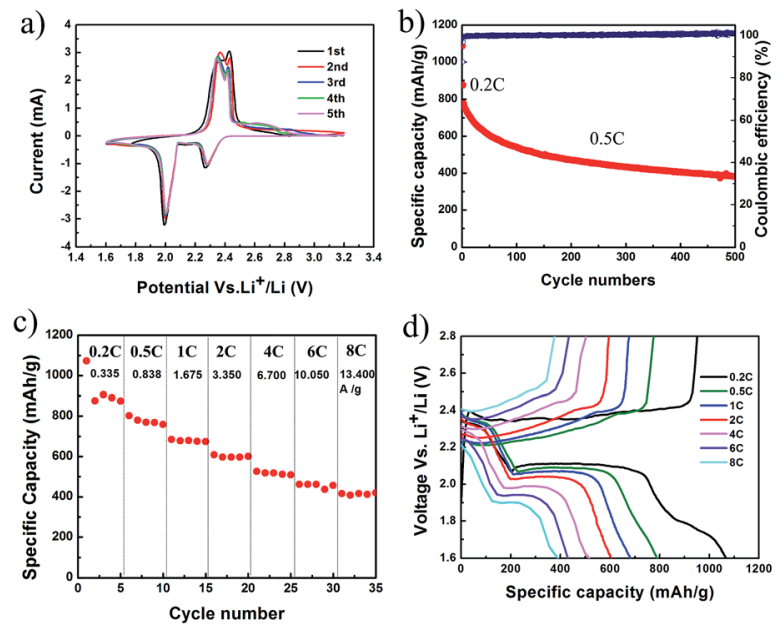

Fig. 5 (a) CV curves of NGP/S at a scan rate of $0.1 \mathrm{mV} \mathrm{s}^{-1}$. (b) Cycling performance, (c) corresponding rate performance, (d) galvanostatic charge and discharge profiles at different current densities of NGP/S.

to the transformations of lithium sulfide to long-chain polysulfide and finally to sulfur. ${ }^{2}$ The repeatable peak position and reaction current suggest the efficient entrapment of polysulfide. The cycling performance of NGP/S is presented in Fig. $5 \mathrm{~b}$. The first two cycles were tested at $0.2 \mathrm{C}$ for activation, and the following cycles were test at $0.5 \mathrm{C}$. The initial discharge capacity reaches a high value of $1087 \mathrm{~mA} \mathrm{~h} \mathrm{~g}^{-1}\left(492 \mathrm{~mA} \mathrm{~h} \mathrm{~cm}^{-3}\right)$, suggesting $65 \%$ utilization of sulfur at $0.2 \mathrm{C}$. The discharge capacity maintains at $400 \mathrm{~mA} \mathrm{~h} \mathrm{~g}^{-1}\left(180 \mathrm{~mA} \mathrm{~h} \mathrm{~cm}^{-3}\right)$ after 500 cycles with a corresponding coulombic efficiency nearly $100 \%$. The capacity loss may firstly come from the excessive sulfur on the surface or gaps between particles. Thus, the excessive sulfur could not be confined by the graphene with nanoshells, which leads to the quick capacity loss in first 100 cycles but a much slower capacity degradation after 100 cycles.

More importantly, NGP/S cathode presents a surprising ultra-high rate capability. As shown in Fig. $5 \mathrm{c}$, with increasing current density from $0.335 \mathrm{~A} \mathrm{~g}^{-1}$ to $10.05 \mathrm{~A} \mathrm{~g}^{-1}$, the specific discharge capacities at C-rate of $0.2,0.5,1,2,4,6 \mathrm{C}$ are about $1070,800,680,600,520,460 \mathrm{~mA} \mathrm{~h} \mathrm{~g}^{-1}$, respectively. Finally, the discharge capacity at $8 \mathrm{C}\left(13.4 \mathrm{~A} \mathrm{~g}^{-1}\right)$ is still as high as $415 \mathrm{~mA} \mathrm{~h}$ $\mathrm{g}^{-1}$, which means that a full discharge or charge process can be completed in about 2 minutes. Compared to similar carbon structures, the rate capability in this unique graphene/sulfur structure was much better. ${ }^{41-43}$ For example, with sulfur/ graphitic hollow carbon sphere nano-composite as a cathode material, $425 \mathrm{~mA} \mathrm{~h} \mathrm{~g}^{-1}$ at $3 \mathrm{C}$ was presented with the same sulfur content about $60 \%$ and specific capacity under higher current density was not tested. ${ }^{41}$ Confining sulfur in double-shelled hollow carbon spheres, specific capacity of $350 \mathrm{~mA} \mathrm{~h} \mathrm{~g}{ }^{-1}$ was obtained at $1 \mathrm{C} .^{42}$ In the meantime, as shown in Fig. $5 \mathrm{~d}$, all the discharge/charge curves kept clear and steady. The two-step galvanostatic discharge profiles were still maintained at such high current density of $13.4 \mathrm{~A} \mathrm{~g}^{-1}$, and high discharge voltage plateau is kept at $2.15 \mathrm{~V}$ and $1.90 \mathrm{~V}$ respectively, better than some other graphene or porous carbon based structures. For
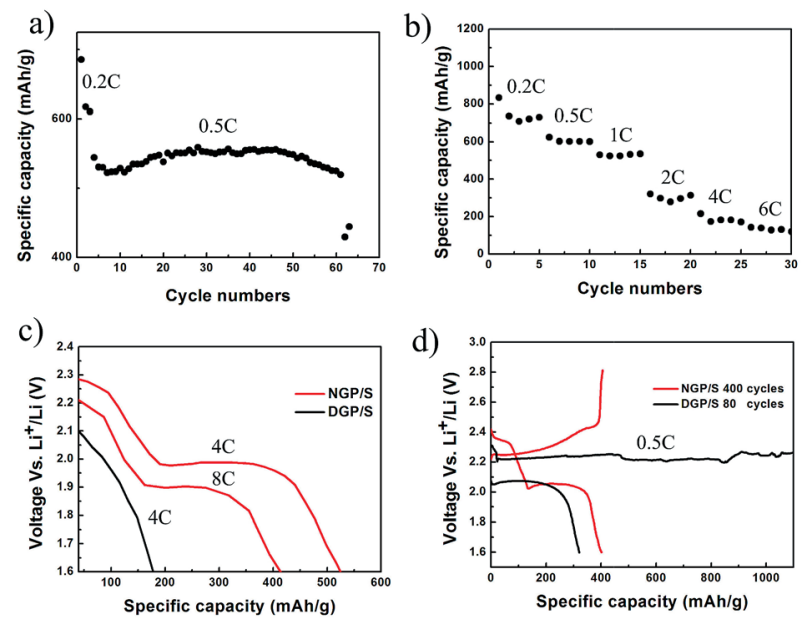

Fig. 6 (a) Cycling performance and (b) rate performance of DGP/S. (c) Discharge profiles of NGP/S and DGP/S at different current density. (d) Charge and discharge profiles of GP at 50th cycle and NGP at 300th cycle.

example, using graphene nanoshell as sulfur host material, the significant slope disappeared at $5 \mathrm{C}$ and the second discharge voltage plateau decreased to $1.5 \mathrm{~V} \cdot{ }^{39}$ Lower discharge voltage plateau and notable slope was also observed with graphenebased layered porous carbon as cathode. ${ }^{44}$

The well designed nanoporous structure was crucial to the long cycling performance and excellent rate performance. As a contrast, the DGP/S exhibits a lower discharge capacity of 685 $\mathrm{mA} \mathrm{h} \mathrm{g}{ }^{-1}$ at the initial cycle and the discharge capacity decreased rapidly after 60 cycles, as shown in Fig. 6a. Meanwhile, the DGP/S has a much worse rate performance, as shown in Fig. 6b. The discharge capacity of DGP/S has a fast decay at 2C and the value declined to $100 \mathrm{~mA} \mathrm{~h} \mathrm{~g}{ }^{-1}$ when current density increases to 6C. As shown in Fig. 6c, the voltage of DGP/S decays rapidly and the corresponding plateau disappears at the current density of $4 \mathrm{C}$, in sharp contrast, it is still well maintained at a much higher current density of $13.4 \mathrm{~A} \mathrm{~g}^{-1}$ in NGP/S. As shown in Fig. 6d, severe overcharge of DGP/S can be observed after 50 cycles, suggesting that the dissolved polysulfide causes redox shuttle between GP/S cathode and Li anode. This phenomenon suggests that polysulfide generated in DGP/S electrode can diffuse to $\mathrm{Li}$ anode to be reduced and then diffuse back to cathode to be oxidized again, and hence lead to endless reaction while the cut-off voltage can't be reached. The charge capacity of several thousand $\mathrm{mA} \mathrm{h} \mathrm{g}{ }^{-1}$ will be achieved if the cut-off capacity was not settled in the test program. However, such phenomenon can be eliminated in NGP/S.

The absence of pore to accommodate sulfur led to low utilization of sulfur and serious dissolution of polysulfide, which resulted in much lower initial discharge capacity and rate performance, bad cycling performance and severe over-charge mentioned above. In contrast, excellent performances were observed in NGP/S due to the successful package of sulfur in NGP and efficient entrapment of polysulfide during cycling process, the efficient electron and ion transfer path constructed by the interconnected few-layer graphene shell inside the NGP. 


\section{Conclusions}

In conclusion, nanoporous graphene powder with high quantity was successfully prepared by a facile spray drying and subsequently CVD process. The NGP powder provided a wellconnected electrical conducting network and also numerous pores for sulfur accommodation which was significantly good for entrapping the dissolution of intermediate polysulfide, facilitating efficient electrical transport and alleviating the volume expansion. The specific discharge capacity of about 400 $\mathrm{mA} \mathrm{h} \mathrm{g} \mathrm{g}^{-1}$ and coulombic efficiency near $100 \%$ were obtained after a long cycling of 500 cycles. More importantly, the specific discharge capacity at high current density of $13.4 \mathrm{~A} \mathrm{~g}^{-1}$ was as high as $415 \mathrm{~mA} \mathrm{~h} \mathrm{~g}^{-1}$. This work paves a facile and universal way to build nanoporous graphene powder with high quality and high quantity which would be very promising for battery, supercapacitor, catalysis and other fields.

\section{Acknowledgements}

This work is supported by the National Natural Science Foundation of China (51402322) and the Natural Science Foundation of Zhejiang Province (LY13A040005).

\section{References}

1 J. Brueckner, S. Thieme, H. T. Grossmann, S. Doerfler, H. Althues and S. Kaskel, J. Power Sources, 2014, 268, 82.

2 S. S. Zhang, J. Power Sources, 2013, 231, 153.

3 A. Manthiram, Y. Fu and Y.-S. Su, Acc. Chem. Res., 2013, 46, 1125.

4 S. Evers and L. F. Nazar, Acc. Chem. Res., 2013, 46, 1135.

5 X. Ji, K. T. Lee and L. F. Nazar, Nat. Mater., 2009, 8, 500.

6 Z. Li, Y. Jiang, L. Yuan, Z. Yi, C. Wu, Y. Liu, P. Strasser and Y. Huang, ACS Nano, 2014, 8, 9295.

7 S. Xin, L. Gu, N. H. Zhao, Y. X. Yin, L. J. Zhou, Y. G. Guo and L. J. Wan, J. Am. Chem. Soc., 2012, 134, 18510.

8 C. Zhao, L. Liu, H. Zhao, A. Krall, Z. Wen, J. Chen, P. Hurley, J. Jiang and Y. Li, Nanoscale, 2014, 6, 882.

9 Z. Lyu, D. Xu, L. Yang, R. Che, R. Feng, J. Zhao, Y. Li, Q. Wu, X. Wang and Z. Hu, Nano Energy, 2015, 12, 657.

10 P. Strubel, S. Thieme, T. Biemelt, A. Helmer, M. Oschatz, J. Brueckner, H. Althues and S. Kaskel, Adv. Funct. Mater., 2015, 25, 287.

11 S. Liu, K. Xie, Z. Chen, Y. Li, X. Hong, J. Xu, L. Zhou, J. Yuan and C. Zheng, J. Mater. Chem. A, 2015, 3, 11395.

12 G. Zheng, Y. Yang, J. J. Cha, S. S. Hong and Y. Cui, Nano Lett., 2011, 11, 4462.

13 Z. Li, J. Zhang and X. W. Lou, Angew. Chem., Int. Ed., 2015, 54, 12886.

14 L. Qie and A. Manthiram, Adv. Mater., 2015, 27, 1694.

15 L. Sun, W. Kong, Y. Jiang, H. Wu, K. Jiang, J. Wang and S. Fan, J. Mater. Chem. A, 2015, 3, 5305.

16 Z. Yuan, H.-J. Peng, J.-Q. Huang, X.-Y. Liu, D.-W. Wang, X.-B. Cheng and Q. Zhang, Adv. Funct. Mater., 2014, 24, 6105.

17 Y. Zhao, W. Wu, J. Li, Z. Xu and L. Guan, Adv. Mater., 2014, 26, 5113.
18 Z. Li, J. T. Zhang, Y. M. Chen, J. Li and X. W. Lou, Nat. Commun., 2015, 6, 8850.

19 Y. Ma, H. Zhang, B. Wu, M. Wang, X. Li and H. Zhang, Sci. Rep., 2015, 5, 14949.

20 Q. Sun, X. Fang, W. Weng, J. Deng, P. Chen, J. Ren, G. Guan, M. Wang and H. Peng, Angew. Chem., Int. Ed., 2015, 54, 10539.

21 C. Zu, L. Li, L. Qie and A. Manthiram, J. Power Sources, 2015, 284, 60.

22 G. He, L. Li and A. Manthiram, J. Mater. Chem. A, 2015, 3, 14750.

23 C. Xu, Y. Wu, X. Zhao, X. Wang, G. Du, J. Zhang and J. Tu, J. Power Sources, 2015, 275, 22.

24 Y. Wu, C. Xu, J. Guo, Q. Su, G. Du and J. Zhang, Mater. Lett., 2014, 137, 277.

25 Y. Liu, J. Guo, J. Zhang, Q. Su and G. Du, Appl. Surf. Sci., 2015, 324, 399.

26 J. Zhang, Z. Dong, X. Wang, X. Zhao, J. Tu, Q. Su and G. Du, J. Power Sources, 2014, 270, 1.

27 H. Chen, C. Wang, Y. Dai, S. Qiu, J. Yang, W. Lu and L. Chen, Nano Lett., 2015, 15, 5443.

28 H. Li, X. Yang, X. Wang, M. Liu, F. Ye, J. Wang, Y. Qiu, W. Li and Y. Zhang, Nano Energy, 2015, 12, 468.

29 G. Zhou, L.-C. Yin, D.-W. Wang, L. Li, S. Pei, I. R. Gentle, F. Li and H.-M. Cheng, ACS Nano, 2013, 7, 5367.

30 S. Lu, Y. Chen, X. Wu, Z. Wang and Y. Li, Sci. Rep., 2014, 4, 4629.

31 X. Feng, M. K. Song, W. C. Stolte, D. Gardenghi, D. Zhang, X. Sun, J. Zhu, E. J. Cairns and J. Guo, Phys. Chem. Chem. Phys., 2014, 16, 16931.

32 Z. Chen, W. Ren, L. Gao, B. Liu, S. Pei and H.-M. Cheng, Nat. Mater., 2011, 10, 424.

33 Y. He, W. Chen, X. Li, Z. Zhang, J. Fu, C. Zhao and E. Xie, ACS Nano, 2013, 7, 174.

34 H. Ji, L. Zhang, M. T. Pettes, H. Li, S. Chen, L. Shi, R. Piner and R. S. Ruoff, Nano Lett., 2012, 12, 2446.

35 G. Zhou, L. Li, C. Ma, S. Wang, Y. Shi, N. Koratkar, W. Ren, F. Li and H.-M. Cheng, Nano Energy, 2015, 11, 356.

36 K. Xi, P. R. Kidambi, R. Chen, C. Gao, X. Peng, C. Ducati, S. Hofmann and R. V. Kumar, Nanoscale, 2014, 6, 5746.

37 T. Wu, H. Shen, L. Sun, B. Cheng, B. Liu and J. Shen, ACS Appl. Mater. Interfaces, 2012, 4, 2041.

38 Z. Wang, P. Li, Y. Chen, J. Liu, H. Tian, J. Zhou, W. Zhang and Y. Li, J. Mater. Chem. C, 2014, 2, 7396.

39 H.-J. Peng, J. Liang, L. Zhu, J.-Q. Huang, X.-B. Cheng, X. Guo, W. Ding, W. Zhu and Q. Zhang, ACS Nano, 2014, 8, 11280.

40 M. Q. Zhao, Q. Zhang, J. Q. Huang, G. L. Tian, J. Q. Nie, H. J. Peng and F. Wei, Nat. Commun., 2014, 5, 3410.

41 E. S. Shin, M. S. Kim, W. I. Cho and S. H. Oh, Nanoscale Res. Lett., 2013, 8, 343.

42 C. Zhang, H. B. Wu, C. Yuan, Z. Guo and X. W. Lou, Angew. Chem., Int. Ed., 2012, 51, 9592.

43 P. Strubel, S. Thieme, T. Biemelt, A. Helmer, M. Oschatz, J. Brückner, H. Althues and S. Kaskel, Adv. Funct. Mater., 2015, 25, 287.

44 D. Wang, A. Fu, H. Li, Y. Wang, P. Guo, J. Liu and X. S. Zhao, J. Power Sources, 2015, 285, 469. 